1 Supporting Information

\title{
2 The origin of air-stability for transition metal oxide 3 cathodes in sodium-ion batteries
}

4 Chenlu $X u^{1}$, Haoran Cai ${ }^{1}$, Qinlong Chen ${ }^{1}$, Xueqian Kong ${ }^{1}$, Huilin Pan ${ }^{1,2 *}$, Yong-Sheng Hu ${ }^{3}$

$5 \quad{ }^{1}$ Department of Chemistry, Zhejiang University, Hangzhou, 310027, China

$6 \quad{ }^{2}$ State Key Laboratory of Clean Energy Utilization, Zhejiang University, Hangzhou 310027,

7 China

$8 \quad{ }^{3}$ Key Laboratory for Renewable Energy, Beijing Key Laboratory for New Energy Materials

9 and Devices, Beijing National Laboratory for Condensed Matter Physics, Institute of Physics,

10 Chinese Academy of Sciences, Beijing 100190, China

11

Corresponding Authors:

13 Huilin Pan (panhuilin@zju.edu.cn)

14

15

16

17

18

19 


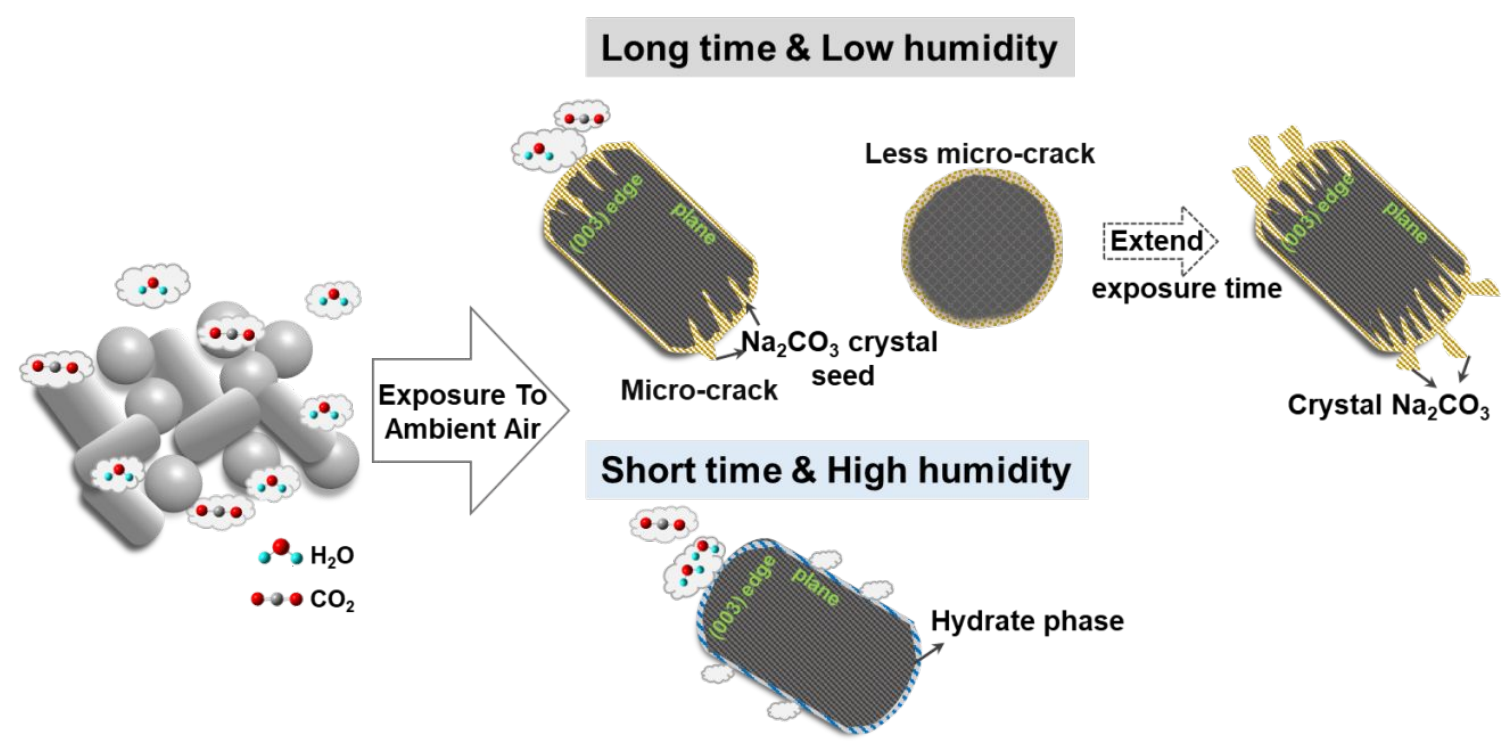

2 Figure S1. Schematic illustration of the reactivity and the deterioration pathway for O3-NFM 3 materials under different atmosphere conditions.
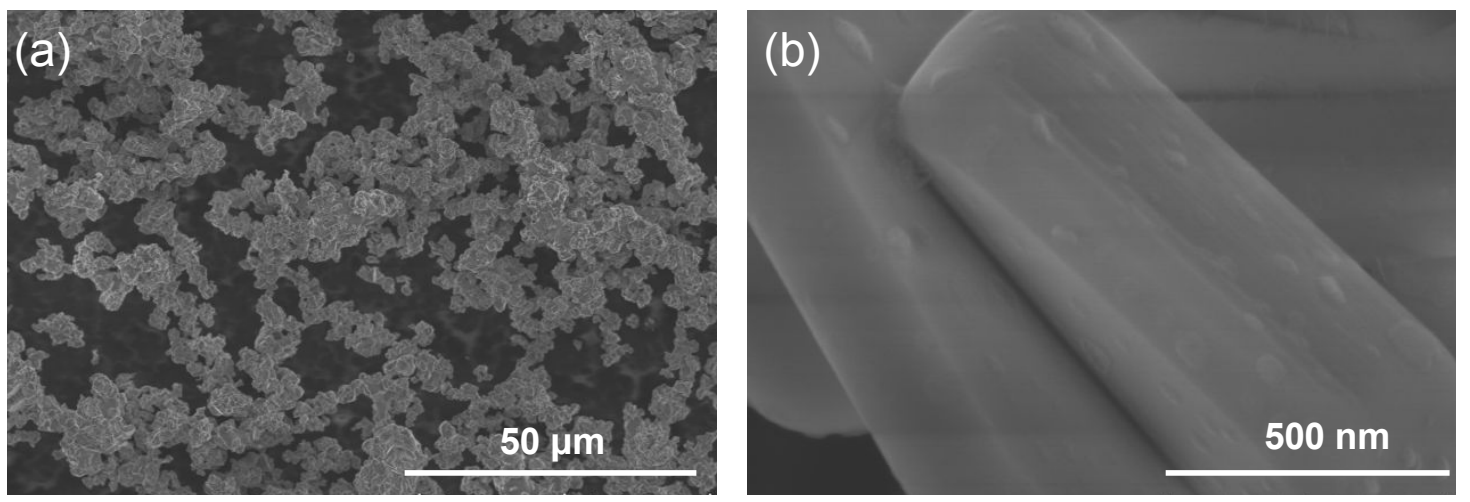

7 Figure S2. The SEM images of the pristine O3-NFM at (a) typical low-magnification and (b)

8 high-magnification.

9 


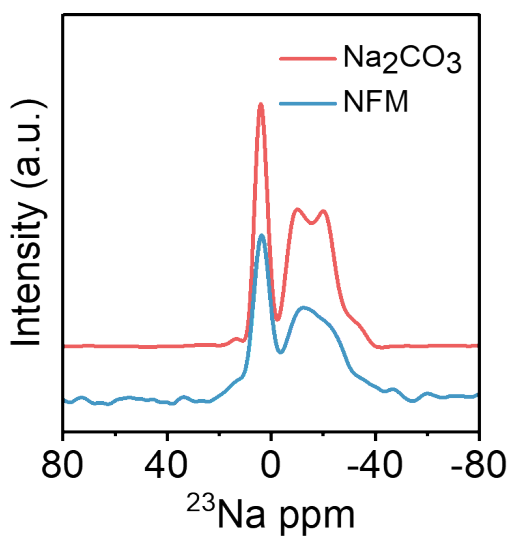

2 Figure S3. ${ }^{23} \mathrm{Na}$ NMR spectra for the pristine O3-NFM and commercial $\mathrm{Na}_{2} \mathrm{CO}_{3}$ powder. 3

4
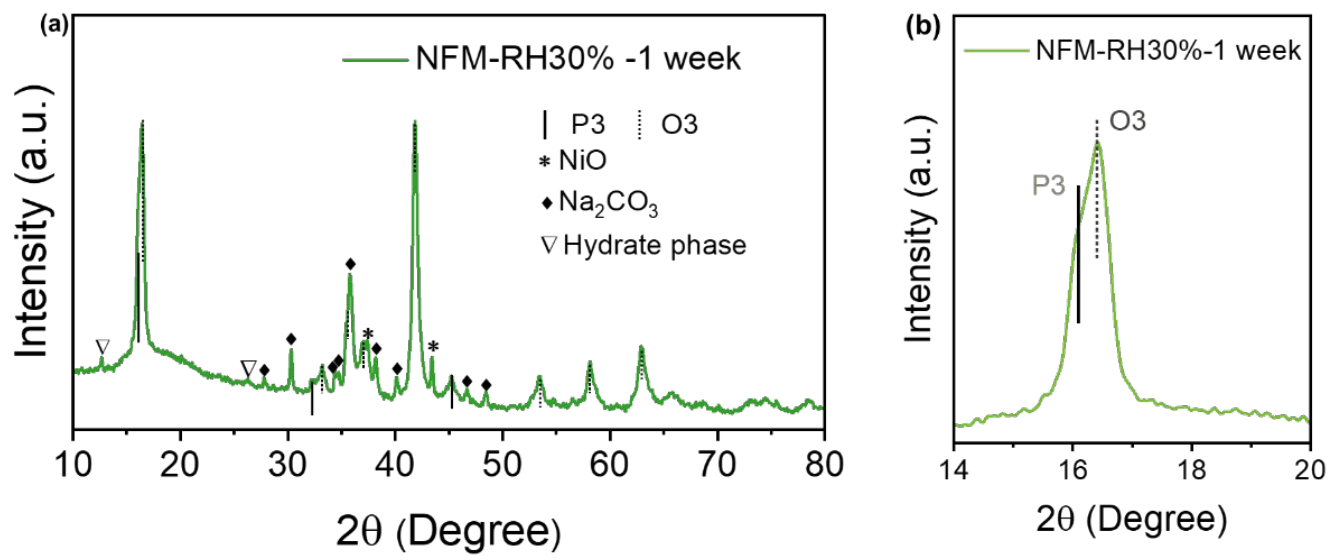

Figure S4. (a) The XRD patterns of O3-NFM stored under 30\% humidity air for one week, the

7 peaks of $\mathrm{Na}_{2} \mathrm{CO}_{3}$ marked by “". (b) The enlarged XRD peaks in the angle range of $14^{\circ} \sim 20^{\circ}$. 8 

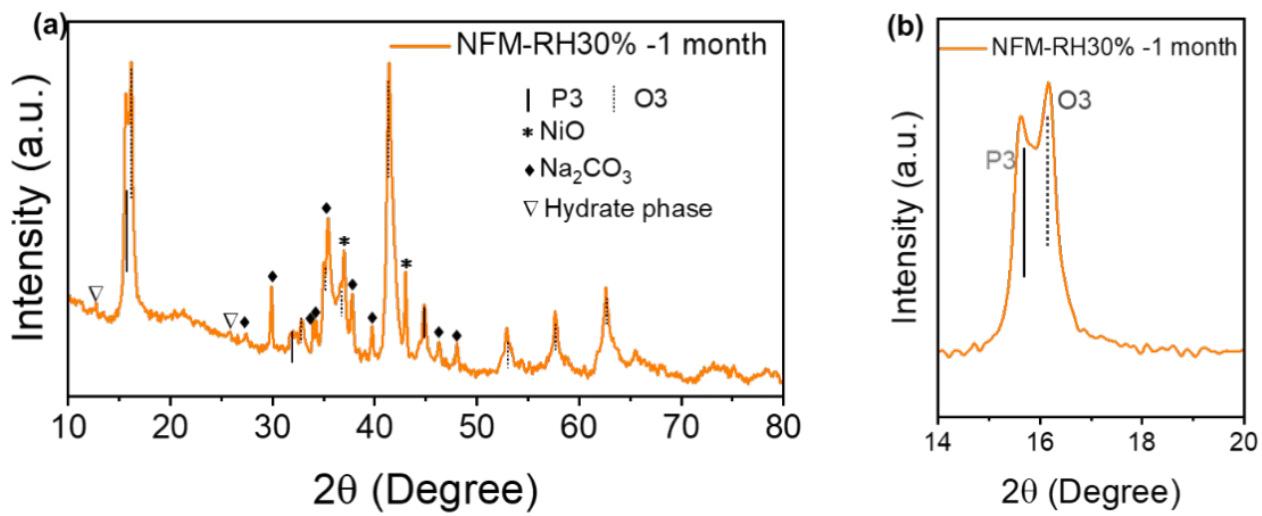

(c)

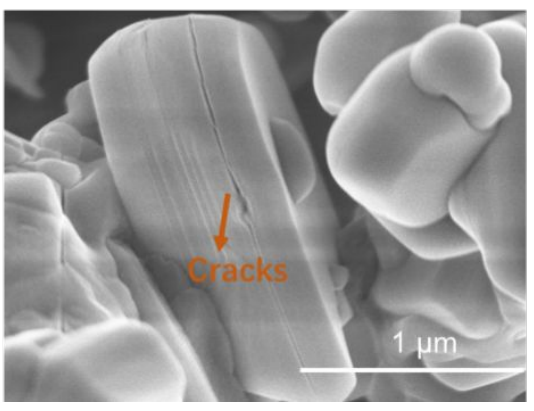

(d)

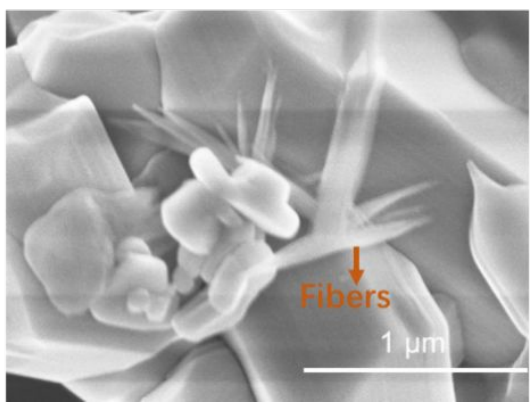

2 Figure S5. (a) The XRD patterns of O3-NFM stored under 30\% humidity air for one month, (b)

3 detail view of the P3 and O3 phase, (c, d) SEM images of NFM-RH30\%-1 month.

4

5

6
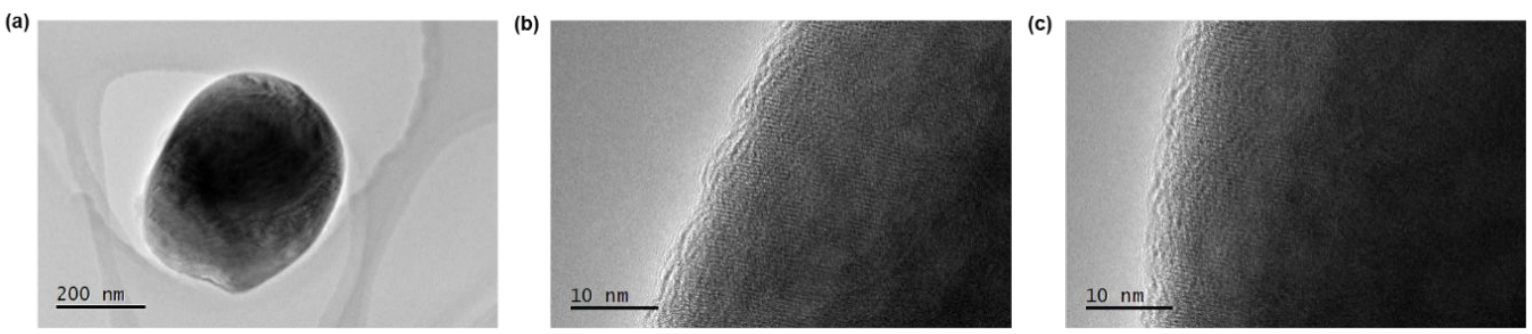

7 Figure S6. HRTEM images of NFM-RH30\%-1week particles (a) with spherical shapes, (b-c)

8 with the smooth edges, showing fewer micro-cracks.

9 

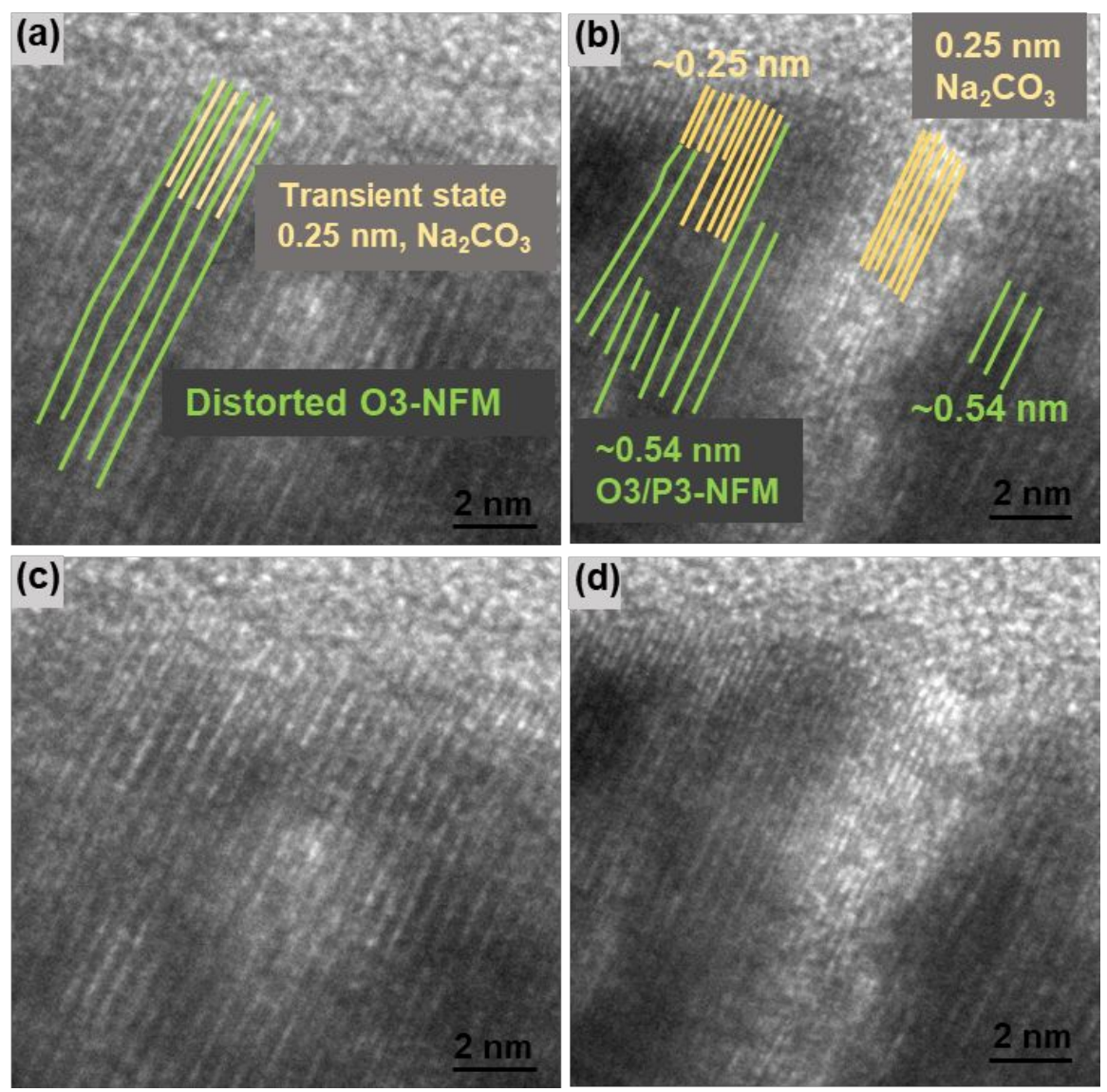

2 Figure S7. The HRTEM images of surface structure changes for (a, b) the NFM-RH30\%-

3 1week sample or (c, d)without marked lines.

4 

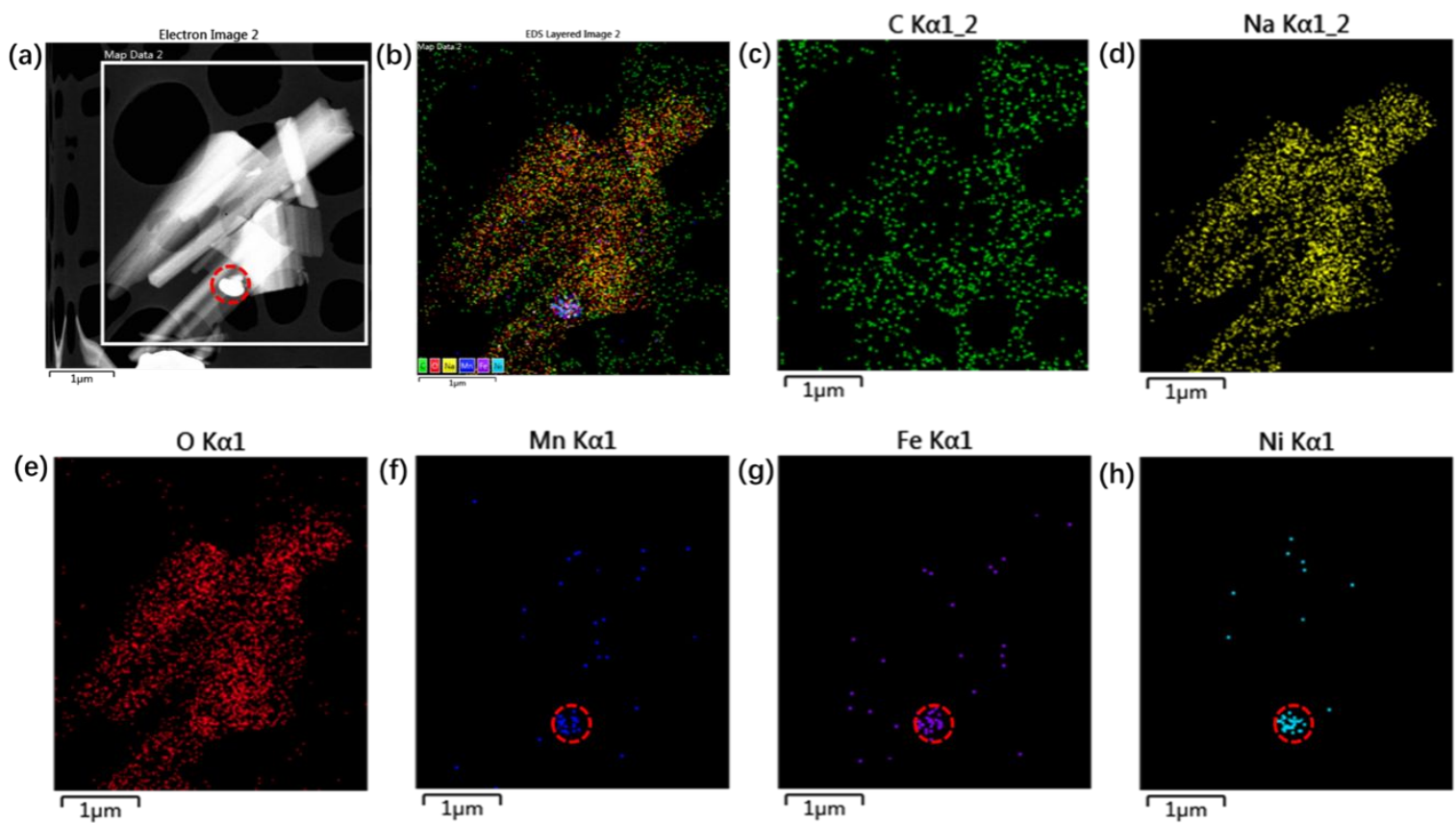

2 Figure S8. (a-b) EDS mapping for the fiber-like particles on the surface of NFM-RH30\%-1

3 month sample. (c-e) $\mathrm{C}, \mathrm{Na}$ and $\mathrm{O}$ elements exhibited similar mapping shapes without obvious

4 signals for (f-g) $\mathrm{Mn}, \mathrm{Fe}, \mathrm{Ni}$, indicating the formation of $\mathrm{Na}_{2} \mathrm{CO}_{3}$ particles on the NFM-RH30\%

51 month sample.

6

7

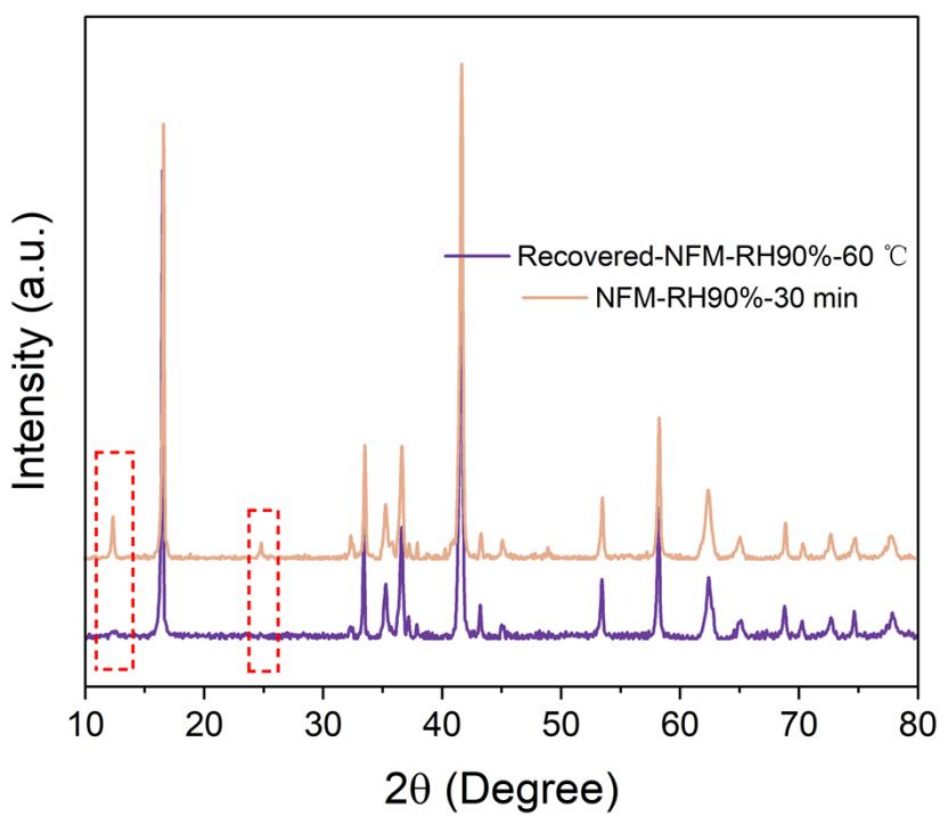

9 Figure S9. The hydrate NFM-RH90\%-30min after heat treatment at $60{ }^{\circ} \mathrm{C}$. 

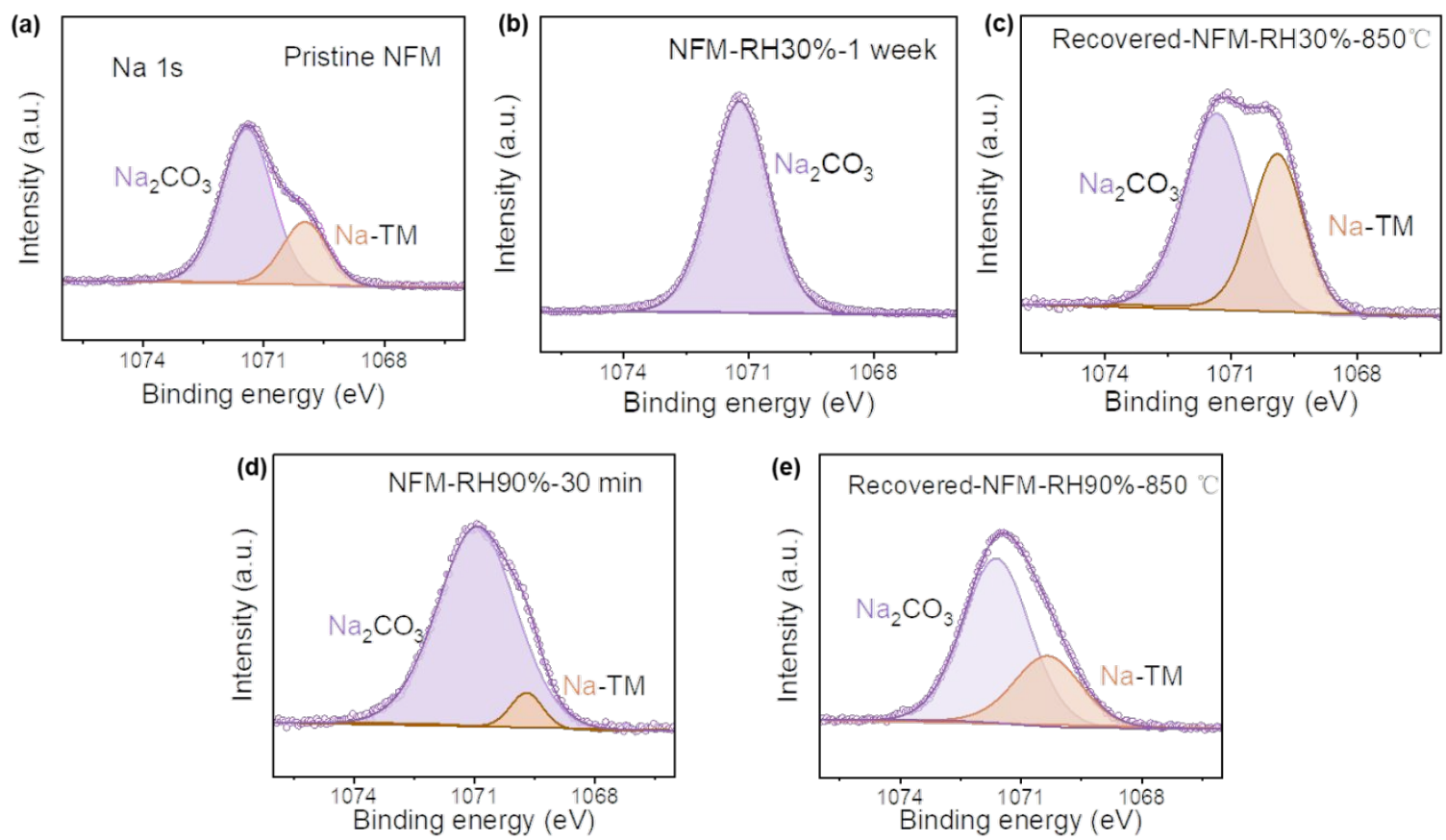

2 Figure S10. XPS spectra of the Na 1s spectra for (a) pristine NFM, (b) NFM-RH30\%-1week,

(c) Recovered-NFM-RH30\%-850 ${ }^{\circ} \mathrm{C}$, (d) NFM-RH90\%-30 min, and (e) Recovered-NFM-

$4 \quad \mathrm{RH} 90 \%-850^{\circ} \mathrm{C}$.
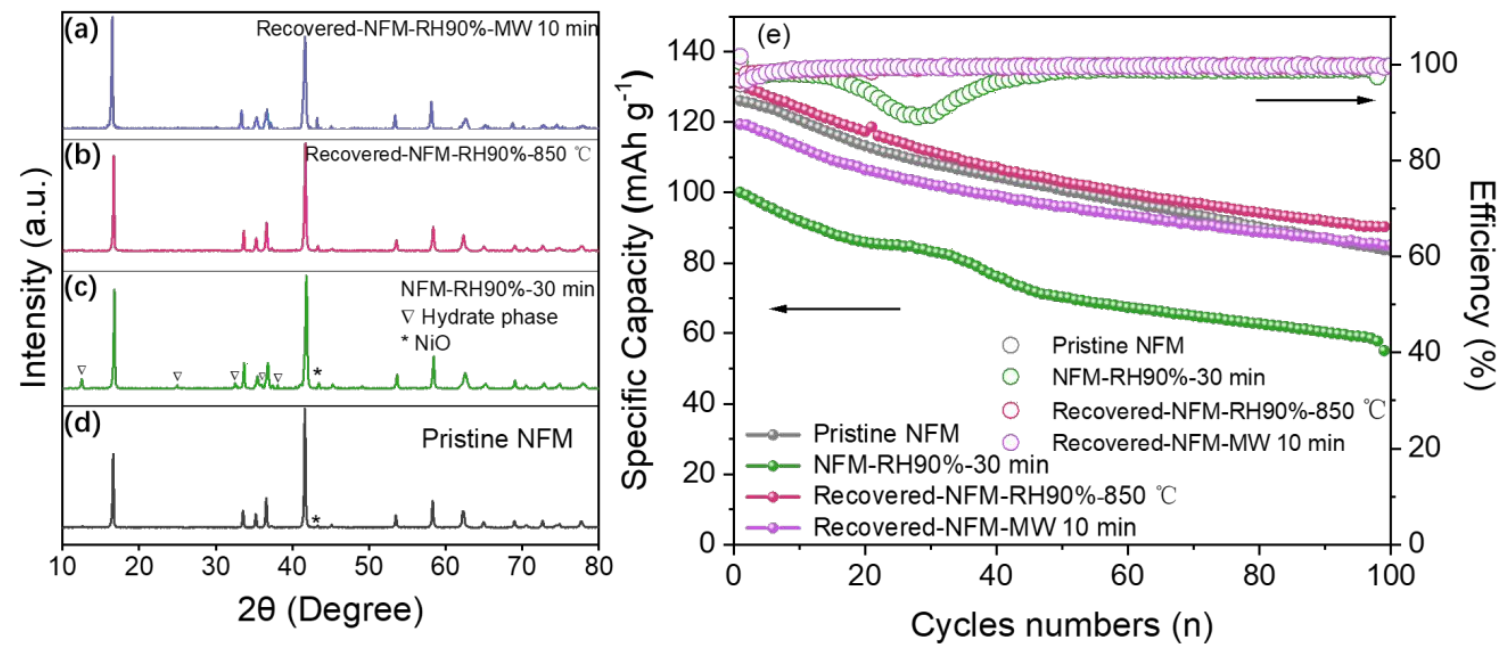

8 Figure S11. XRD patterns of (a) Recovered-NFM-RH90\%-MW 10 min (micro-waving heating

9 for 10 minutes), (b) Recovered-NFM-RH90\%-850 ${ }^{\circ} \mathrm{C}$ (heating for an hour at $850{ }^{\circ} \mathrm{C}$ ), (c) NFM-

RH90\%-30 min, (d) pristine NFM, (e) comparison of the electrochemistry performance for the 
NFM samples.
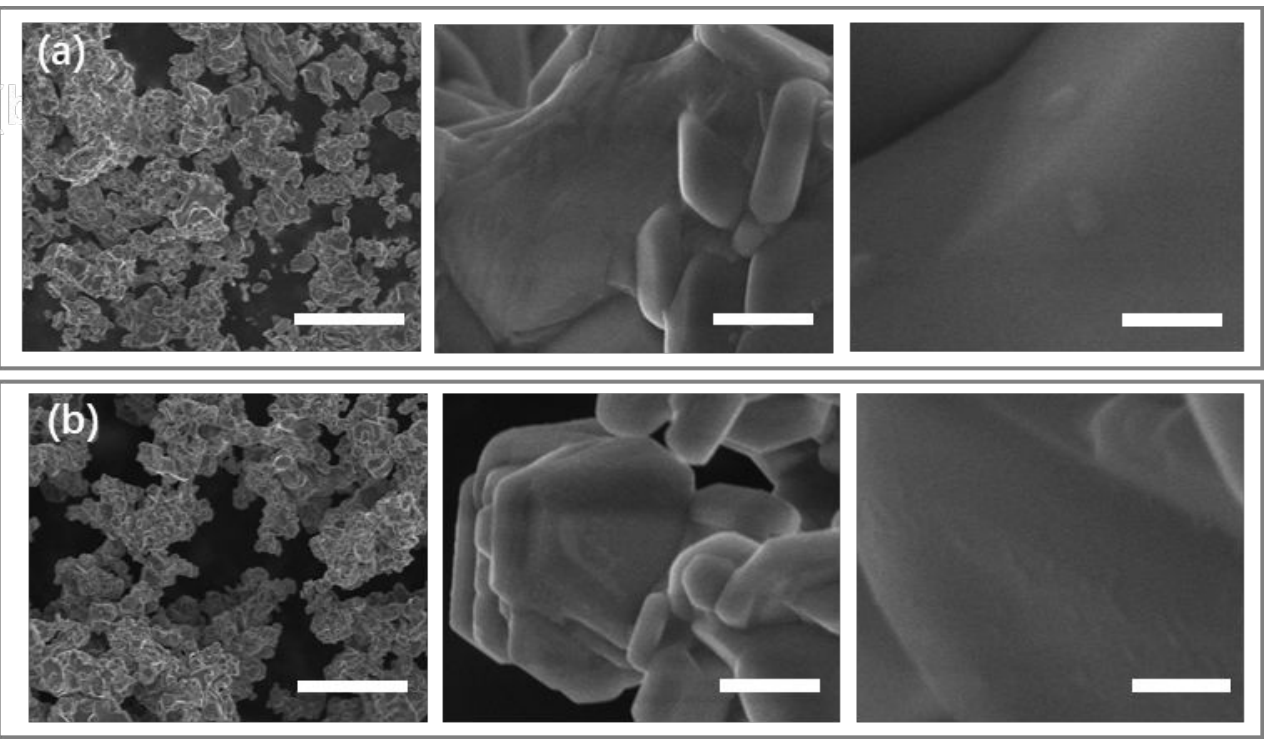

3 Figure S12. The SEM images of the (a) recovered NFM-RH30\%-1week, and (b) recovered

4 NFM-RH90\%-30min samples at $850{ }^{\circ} \mathrm{C}$ at different magnifications. Scale bar: $1 \mu \mathrm{m}$ (left

5 column); $500 \mathrm{~nm}$ (middle column); $200 \mathrm{~nm}$ (right column).

6

7
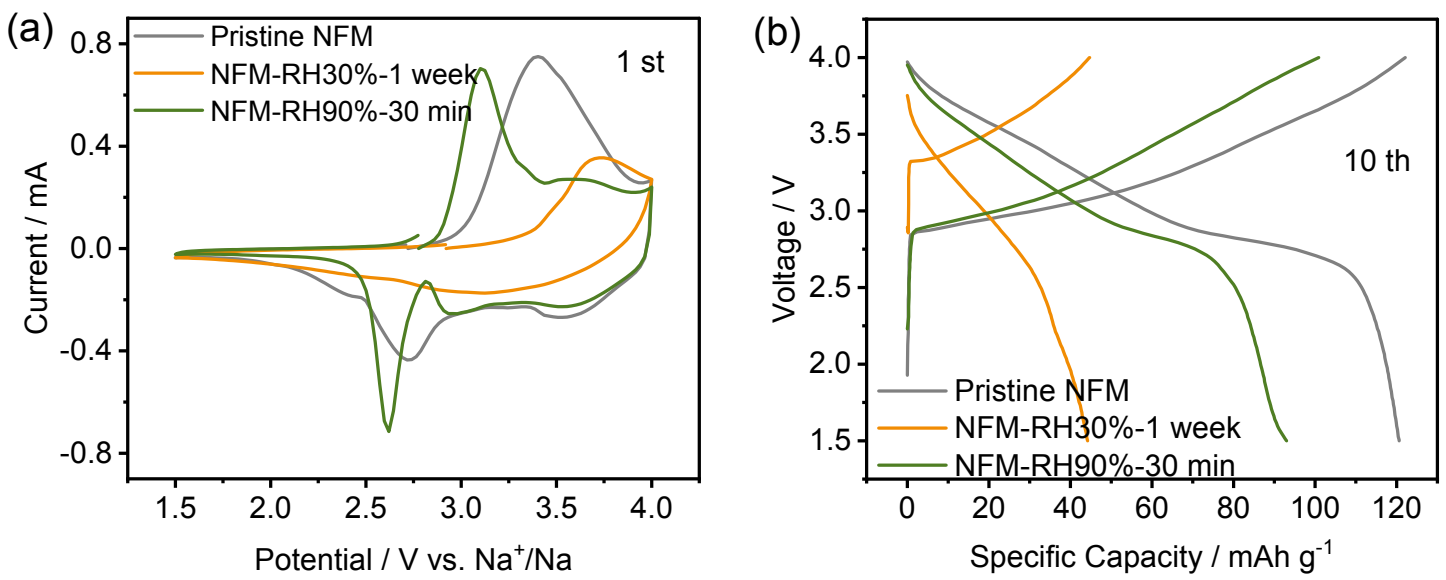

9 Figure S13. (a) CV curves and (b) charge-discharge curves for the pristine NFM, NFM-

10 RH30\%-1week and NFM-RH90\%-30min samples, respectively (charge-discharge C rate:

$11 \quad 0.5 \mathrm{C})$. 
(a)

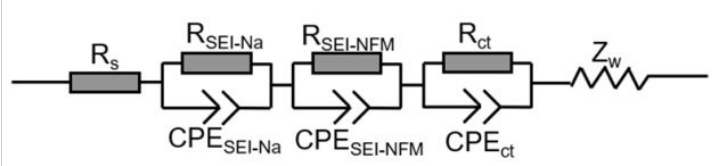

(b)

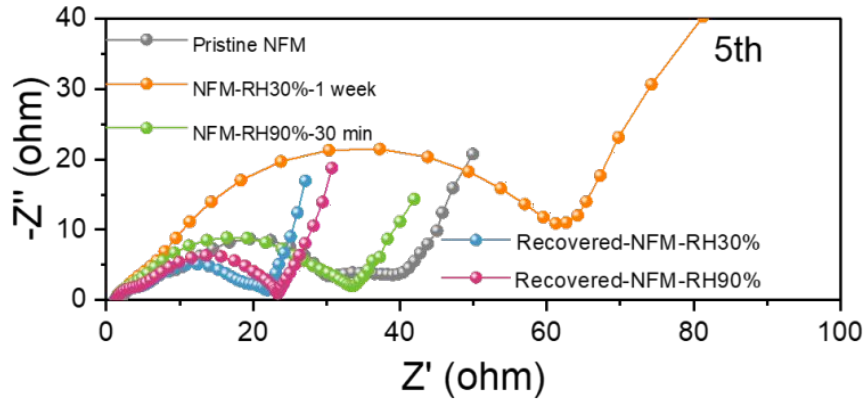

(c)

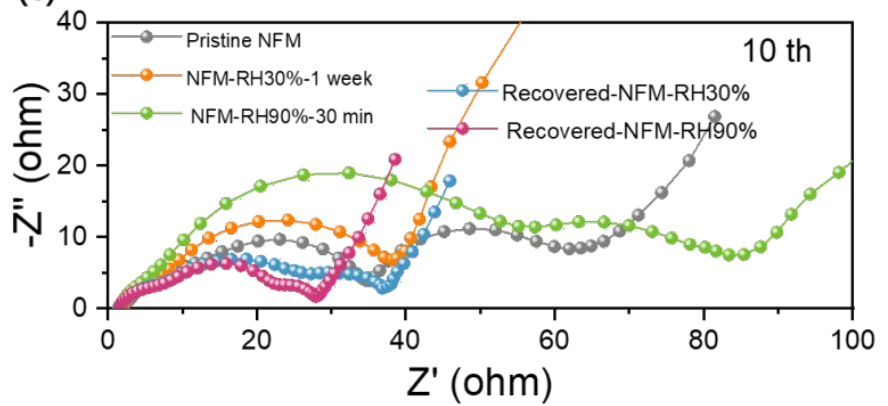

3 Figure S14. (a) The equivalent circuit of EIS spectrum, (b, c) Enlarged EIS spectra for the

4 pristine, air-exposed and recovered NFM samples in the $5^{\text {th }}$ and $10^{\text {th }}$ cycles. The Figure S14

5 showed the enlarged plots of the EIS spectra for the degraded O3-NFM and the recovered O3-

6 NMF samples and the equivalent circuit for the EIS spectra. The EIS spectra all showed three

7 consecutive semicircles in the high and medium frequency range, and a linear slope in the low

8 frequency. The three semicircles from high to low frequency could be ascribed to the solid 9 electrolyte interface (SEI) formation on the NFM and $\mathrm{Na}$ counter electrodes $\left(\mathrm{R}_{\mathrm{SEI}-\mathrm{Na}}\right)$, the 10 transportation of $\mathrm{Na}^{+}$ions inside the NFM electrodes ( $\left.\mathrm{R}_{\mathrm{SEI}-\mathrm{NFM}}\right)$, and the charge transfer process $11\left(\mathrm{R}_{\mathrm{ct}}\right)$, respectively .The low frequency slope could be from the Warburg impedance. ${ }^{1,2}$ The 12 NFM-RH30\%-1week electrode (yellow curve in Figure S14b) showed the largest impedance 13 for each electrochemical process, indicating the formation of $\mathrm{Na}_{2} \mathrm{CO}_{3}$ and microcracks in the 14 crystal structure of NFM could impede the effective charge transfer in the NFM particles. This 15 is consistent with the low Na storage capacity and increased overpotential of the NFM-RH30\%16 1week sample as discussed in Figure 5g. The NFM-RH90\%-30min sample showed almost 17 overlapped EIS curves with the pristine O3-NFM, suggesting the uptake of $\mathrm{H}_{2} \mathrm{O}$ molecules 
1 would not directly affect the charge transfer kinetics. However, the stability of hydrate NFM

2 could be challenging during cycling. It was evidenced by the quickly doubled impedance from

3 the 5th to 10th cycle for the NFM-RH90\%-30min sample (green curve in Figure 14b-14c).

4 Interestingly, the degraded NFM samples after recovery (blue and pink curves) both showed 5 reduced impedance with respect to the pristine O3-NFM (grey curve in Figure 14b-14c). These

6 results suggest that the recovered NFM samples could benefit fast charge transfer and the

7 formation of stable electrode-electrolyte interface.

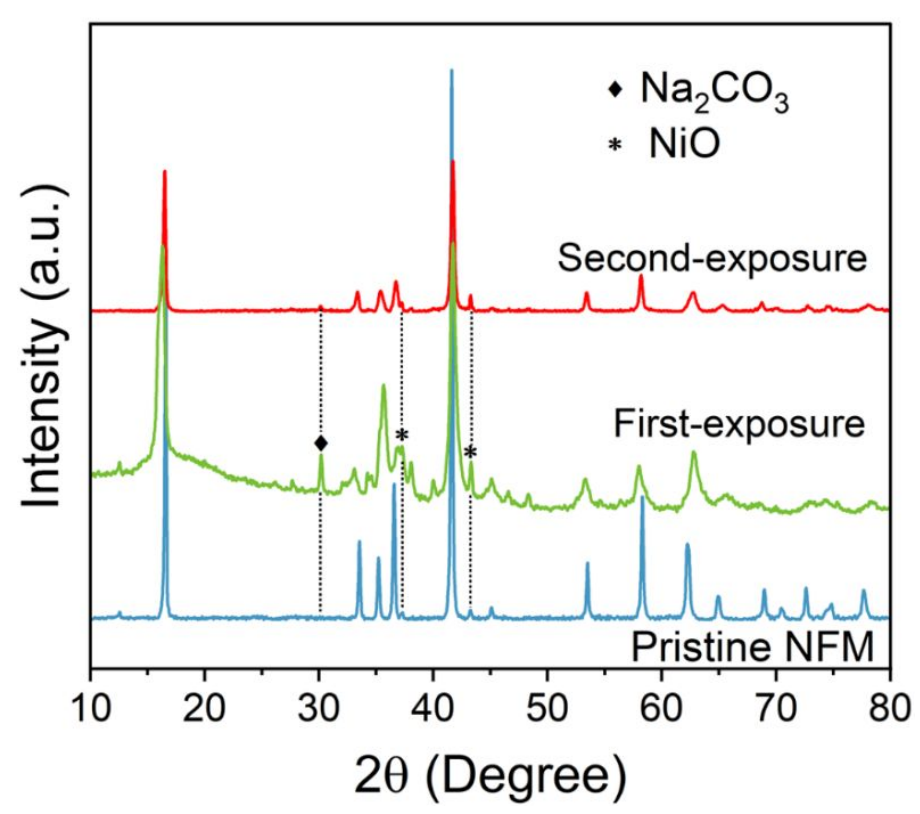

11 Figure S15. XRD patterns for different NFM samples exposed to relative air humidity of $30 \%$

12 for one week. Blue line: pristine NFM without exposure. Green line: pristine NMF after air

13 exposure. Red line: Recovered NFM sample exposed to relative air humidity of $30 \%$ for one 14 week again.

\section{Reference:}


1 1. Bredar, A. R. C.; Chown, A. L.; Burton, A. R.; Farnum, B. H., Electrochemical 2 Impedance Spectroscopy of Metal Oxide Electrodes for Energy Applications. ACS Applied 3 Energy Materials 2020, 3 (1), 66-98.

4 2. Zhang, W.; Richter, F. H.; Culver, S. P.; Leichtweiss, T.; Lozano, J. G.; Dietrich, C.; 5 Bruce, P. G.; Zeier, W. G.; Janek, J., Degradation Mechanisms at the $\mathrm{Li}_{10} \mathrm{GeP}_{2} \mathrm{~S}_{12} / \mathrm{LiCoO}_{2}$ 6 Cathode Interface in an All-Solid-State Lithium-Ion Battery. ACS Applied Materials \& 7 Interfaces 2018, 10 (26), 22226-22236.

8 